\title{
Rolf Knieper \\ Die Freiheiten des Willens, \\ der Person und des Eigentums in der Metaphysik des Zivilrechts
}

\begin{abstract}
Der Beitrag versucht, die normativen Schablonen der privatrechtlichen Freibeiten des Willens, des Rechtssubjekts und des Eigentums aufzuspüren und von naturrechtlichen Vorverständnissen zugunsten des positiven Rechts zu reinigen. Daraus ergeben sich unmittelbare Konsequenzen für eine zureichende Erklärung juristischer Figuren und rechtspolitischer Forderungen. Er baut auf einem Vortrag auf, den Verfasser an der Waseda Universität in Tokio gebalten hat.
\end{abstract}

\section{Ein Eingangsbeispiel}

Ein $»$ man of ripe years and of sound mind, acting freely and with his eyes open ${ }^{\mathrm{I}}$ entschließt sich, aus seiner Sammlung ein Gemälde zu verkaufen, um es gelegentlich durch ein anderes zu ersetzen. Nichts hält ihn von der Realisierung des Vorhabens ab, nichts zwingt ihn dazu, keine Not, keine Eigentumsschranken, kein Gewerbe, kein staatliches oder religiöses Gebot. Er wendet sich an einen Kunsthändler, verhandelt mit ihm über den Preis unter Würdigung der Qualität des Bildes, die Lieferbedingungen, den Ort, den Zeitpunkt, die Zahlungsmodalitäten. Nichts prädestiniert den Inhalt des Vertrages, keine Tarife, kein Marktpreis, keine Listen, keine Allgemeinen Geschäftsbedingungen.

Es ist eine idyllische Situation und eine, auf welche die seit dem i 8. Jahrhundert begonnene, im I9. und frühen 20. Jahrhundert stark anschwellende und bis heute in manchen Orten und Schriften immer noch hartnäckig verteidigte Auffassung vollständig zuzutreffen scheint, gemäß der im Vertrag die substantielle Freiheit des Willens des Menschen rein zum Ausdruck kommt: Der reife und kluge Mann kann überlegen, ob er sein Eigentumsrecht an einem Gegenstand auf einen anderen übertragen will und wenn ja, unter welchen Bedingungen er dazu bereit ist.

Er kann stattdessen das Bild auch behalten und weiter betrachten. Falls er sittenstreng ist oder fundamental religiös oder einer starken politischen Überzeugung anhängt und etwa eine bestimmte Rasse oder Klasse für überlegen hält und eine andere verachtet, und wenn ihm auf seinem Bild eine pornographische oder homoerotische Szene auffällt oder er Gott dargestellt sieht, was er für blasphemisch hält, oder etwas zum Beispiel Anti-Germanisches oder Anti-Proletarisches entdeckt, kann er das Bild auch zerstören. Er kann das im Übrigen auch völlig ohne Motiv² tun, ganz ebenso, wie er ein vollständig bewohnbares Haus und Grundstück im Herzen Tokios oder Frankfurts leer stehen lassen, eine

I So die schöne Definition eines wirklich geschäftsfähigen Gentleman durch das englische House of Lords aus dem Jahre I875, zitiert nach Cooke/Oughton, The Common Law of Obligations, 2nd edition, I993, p. 379 . 
Erfindung patentieren, aber nicht nutzen oder ein profitables Unternehmen liquidieren kann.

Das Freiheitspathos des Zivilrechts nimmt die klassische Trias des Eigentumsrechts absolut und ernst und dekretiert, dass der Eigentümer »mit der Sache nach Belieben verfahren und andere von jeder Einwirkung ausschließen« kann. Diese Formulierung einer umfassenden, durch individuelle oder gesellschaftspolitische Zwecke nicht eingeschränkten Befugnis des $\$ 903$ BGB wiederholt sich in allen naturrechtlich inspirierten Gesetzbüchern, aber auch im case law.

\section{Die Freibeit des Willens}

Sie gibt der Metapher »stat pro ratione voluntas «3 Nahrung. Dieser Schlachtruf der post-pandektistischen Zivilrechtsdogmatik klingt ebenso unverrückbar wie er zwiespältig ist; er lässt sich nämlich sowohl übersetzen als: »der Wille ersetzt die Vernunft« wie: »der Wille steht für die Vernunft«. Er beruft sich auf das Erbe der Aufklärungsphilosophie und der klassischen Pandektistik. Immerhin hatte Hegel formuliert: »Die Freiheit des Willens selbst, als solche, ist Prinzip und substantielle Grundlage allen Rechts«, 4 und Savigny hatte präzisiert: »Das Recht dient der Sittlichkeit, aber nicht indem es ihr Gebot vollzieht, sondern indem es die freye Entfaltung ihrer, jedem einzelnen Willen innewohnenden Kraft sichert «, s während Windscheid, einer der führenden Stichwortgeber des BGB, meint, das Rechtsgeschäft sei eine »auf die Hervorbringung einer rechtlichen Wirkung gerichtete Privatwillenserklärung «. ${ }^{6}$

Das Konzept der Willensherrschaft und -freiheit ist auf ganz verschiedenen Ebenen kritisch hinterfragt worden:

So hat das Bundesverfassungsgericht nach Jahrzehnten der Vorbereitung durch eine sozialdemokratisch-ordoliberal orientierte Literatur zum konsequenzenreichen Verfassungsrechtsgrundsatz erhoben, dass von Vertragsfreiheit nur die Rede sein könne, wenn keiner der Geschäftspartner marktmächtig sei oder wenn ein ungefähres Gleichgewicht an Marktmacht bestehe. Es hat daraus - natürlich, könnte man sagen - nicht die revolutionäre Konsequenz der Zerstörung von Marktmacht gefordert, sondern sozialstaatliche Korrekturen und Kompensation.7 Am treffendsten ist diese Kritik in der Parabel des freien Fuchses im freien Hühnerstall zusammengefasst worden.

So hat Marx gegenüber seinem Lehrer Savigny die Determiniertheit der Sphäre der Warenzirkulation betont, in der sich nur umsetze, was in der determinierenden Produktionssphäre geschehe, ${ }^{8}$ was in gewisser Weise im bomo oeconomicus der ökonomischen Analyse des Rechts seine moderne Verlängerung findet. Es ließe sich sogar sagen, dass in unserem Ausgangsbeispiel problematisch ist, von

2 Die Motive habe ich genannt, um die Problematik nicht auf Individualismus zu verengen: Politisch mächtige soziale Kollektive haben - meistens gegen den Widerstand aus den eigenen Reihen - als Christen, Juden, Muslime, Hindus oder tibetanische oder andere Mönche die Bilder und andere Kunstgegenstände der Nicht-Gläubigen ebenso zerstört wie faschistische oder proletarische Kultusminister dies mit der Kunst der Andersartigen getan haben.

3 Statt aller Flume, Rechtsgeschäft und Privatautonomie, in: Festschrift zum roojährigen Bestehen des deutschen Juristentages, I 960 , S. I 35 ff/ I 4 I.

4 Hegel, Vorlesungen über die Philosophie der Geschichte, I832, zitiert nach: Werke in 20 Bänden, I970, Band 7, S. 524.

5 Savigny, System des heutigen Römischen Rechts, Erster Band, I840, S. 332.

6 Windscheid, Lehrbuch des Pandektenrechts, Dritte Auflage, I887, Erster Band, S. I 87.

7 BVerfGE 89, 2 I $4 \mathrm{ff}$.

8 Marx, Das Kapital - Kritik der politischen Ökonomie, Band I, I867, I. Abschnitt, Zweites Kapitel. 
der Freiheit des Kunsthändlers zu sprechen, da seine Existenz vom Bilderhandel und er dementsprechend materiell vom Vertragsschluss abhängt und er ihn nicht ohne weiteres in Freiheit zurückweisen kann.

So hat von einer ganz anderen, nicht ökonomischen, sondern anthropologischen Warte nun auch die Hirnforschung der Willensfreiheit einen Determinismus entgegengesetzt, der durch neurologische Prozesse bestimmt sei. ${ }^{9}$

All das mögen gewichtige Aussagen sein, und ich komme auf die eine oder andere zurück. Dennoch will ich nicht von ihnen ausgehen, da ich meine, dass selbst wenn man sie insgesamt als richtig unterstellte, die Problematik und der Platz der Privatautonomie im Zivilrecht noch nicht hinreichend analysiert wären. Kehren wir zum Ausgangsbeispiel und dabei zum Bildeigentümer zurück. Wir hatten gesehen, dass er die Idylle des reifen, verständigen und damit im Sinne des Gesetzes freien Mannes ausfüllt. Dennoch begibt er sich dieser Freiheit irgendwann, nachdem er ein Verkaufsangebot gemacht hat. Das scheint der Grundannahme von der Willensfreiheit zu widersprechen. Tatsächlich haben sich verschiedene Gesetze, die sich der Aufklärungsphilosophie und Pandektistik verpflichtet fühlten, schwer getan, den Moment zu bestimmen, von dem an der Anbietende seinen Willen nicht mehr frei äußern konnte. Das ist zum Beispiel der Fall, wenn ihm die Rücknahme oder der Widerruf des Angebots oder gar der Rückzug vom bereits geschlossenen Vertrag verwehrt wird, obwohl er in freier Selbstbestimmung seinen Willen geändert hatte und nicht mehr verkaufen wollte.

Konsequent im Sinne einer durchgängigen Berücksichtigung des individuellen Willens wäre es wohl, den Erklärenden nicht an dem einmal geäußerten Willen festzuhalten und Veränderungen zu ignorieren, sondern an die jeweilige Betätigung Konsequenzen zu knüpfen, den Erklärenden also frei zu lassen, aber ihn die negativen Folgen seines freien Handelns spüren zu lassen. Tatsächlich ist dies die ursprüngliche Konzeption des englischen »assumpsit", gemäß der die Folge einer einseitigen Aufhebung eines Vertrages ein Schadensersatzanspruch ist und nicht ein kontra-voluntaristisches Festhalten an der primären Erfüllungspflicht. Noch heute muss diese gesondert vereinbart werden, um die Pflicht zu einer specific performance zu begründen. ${ }^{\mathrm{I}} \textrm{ }$

Das deutsche und das kontinental-europäische Recht haben schon früh einen anderen Weg eingeschlagen und die Willensfreiheit beschränkt: Die philosophische Vorlage hatte Hegel geliefert, der den subjektiven Willen, die Willkür der Gesinnung, die jederzeit veränderliche Meinung scharf vom geäußerten, durch den Willen einer anderen Person ergriffenen und dadurch gebundenen Willen unterschieden hatte. In der auf einen Anderen orientierten Äußerung des Willens, in der Erklärung, auch der impliziten Handlung vergegenständliche er sich, werde äußerlich und vom Recht erfasst. Mit der Annahme durch den Anderen entstehe der Vertrag und damit »das Recht an sich als ein Gesetztes, seine innere Allgemeinheit als ein Gemeinsames der Willkür und besonderen Willen «. ${ }^{\text {I }}$ Windscheid hatte dies in juristische Sprache übersetzt und den Vertrag dadurch definiert, dass »die Willenserklärung des Anderen ergriffen und festgehalten« werde. ${ }^{\mathrm{I} 2}$ Ein einmal geäußerter Wille wird also bindend, wenn und weil das Gesetz es so will. Damit war positiv-rechtlich die Schwierigkeit überwunden, vor die sich die Anhänger einer heute noch nicht überwundenen Auffassung

9 Vgl. etwa Günther, Hirnforschung und strafrechtlicher Schuldbegriff, in: Kritische Justiz 2006, S. I I 6 ff. Io Vergleiche Cooke/Oughton (Fn .1), S. 8 ff.

I I Hegel, Grundlinien der Philosophie des Rechts, I 82 I (Fn. 4), $\$ 82$ sowie $\iint 78-8$ I, I04, I05.

I 2 Windscheid (Fn. 6), S. I 87. 
gestellt sahen, gemäß der alle Rechtsverhältnisse Willensverhältnisse seien und der Wille frei und ungebunden sei. Diese Annahme kann tatsächlich die praktisch unabweisliche Notwendigkeit einer Bindung von Angeboten, Annahmen und Verträgen theoretisch kaum begründen oder hinterlässt doch - wie Kant es formuliert hat - eine »Verlegenheit «, ${ }^{\mathrm{I} 3}$ da der Willenspurismus sich an der Realität und seinen Zwängen brechen muss.

Wann die Bindung zum Beispiel des Vertragsangebotes einsetzt, haben verschiedene Gesetze unterschiedlich festgelegt. $\int$ I 45 des deutschen BGB legt die Bindungswirkung ebenso weit vor wie das österreichische ABGB (\$ 862). Beide bestimmen, dass das Angebot vor Ablauf der Annahmefrist nicht zurückgenommen werden kann. Demgegenüber ergibt sich aus Art. I328, I 326 und I 335 des italienischen Codice Civile, dass der Widerruf des Antrags bis zum Zugang der Annahme zulässig ist, während das holländische Wetboek ebenfalls die Widerrufbarkeit vorsieht, allerdings nur bis zum Zeitpunkt der Absendung der Annahmeerklärung (Art. 2 I9-6.5.2.2). Das UN-Übereinkommen über Verträge über den Internationalen Warenkauf von I980, dem inzwischen mehr als 65 Staaten beigetreten sind ${ }^{14}$ und das sich bemühen muss, auf verschiedene Rechtskulturen Rücksicht zu nehmen, lässt ebenfalls eine Rücknahme (Art. I s) und einen Widerruf (Art. I6) zu, ohne allerdings ihre rechtlich statuierte Verbindlichkeit zu bezweifeln.

Die Verschiedenartigkeit der rechtlichen Regelungen dokumentiert zum einen, dass die Gesetze es sich mit der Fixierung der Bindung nicht leicht gemacht haben. Sie dokumentiert aber vor allem, dass die Gesetze entscheiden, an welche Merkmale Rechtsfolgen geknüpft werden. Der Wille erzeugt nicht Rechte und Pflichten, sondern er wird wie das Delikt oder andere Handlungen behandelt. In der Sprache der Pandektistik, die wohl der Willensfreiheit verpflichtet war, ohne aber das Verhältnis von privater und staatlicher Kompetenz zu vermischen, gehören Willenserklärung und Vertrag zu den »Thatsachen, mit denen das Recht die Wirkung des Erwerbes oder Verlustes von Rechten verknüpft «. Is

Die ideologische Fixierung der juristischen Dogmatik auf den menschlichen Willen als Rechtsgrund hat zur höchst unbefriedigenden Konsequenz, zu viele für die Praxis selbstverständliche Rechtsfiguren nicht einordnen zu können. Sie muss zu resignierter Hinnahme solcher Praxis, zu Fiktionen oder zu anderen Konstruktionen einer erklärungslosen Theorie Zuflucht nehmen. Es liegt wohl auf der Hand, dass Theorien, die große Bereiche der gesellschaftlichen Praxis nicht erfassen können, kaum überzeugen können.

Als Beispiele des unbefriedigenden Standes der Theorie können wiederum ganz unspektakuläre Erscheinungen herangezogen werden, von denen ich drei benennen will. ${ }^{16}$

So ist behauptet worden, dass die gesetzliche Einführung eines zeitlich begrenzten Rechts zum Widerruf von Verträgen durch Verbraucher an den Grundfesten des Vertragsrechts rütteln. ${ }^{17}$ In Wahrheit hat das Gesetz nichts weiter getan als die Bindungswirkung einer Willenserklärung nach hinten zu verschieben.

Andererseits ist behauptet worden, es werde ein Paradigmenwechsel des Schuldrechts vollzogen, wenn das Gesetz eine öffentliche Erklärung an einen unbe-

I 3 Kant, Die Metaphysik der Sitten, zweite Auflage 1798 (zitiert nach der Werke-Ausgabe von Weischedel, Band IV), S. 384 .

I4 Allerdings gehört Japan bisher nicht zu den Mitgliedstaaten.

is Puchta, Pandekten, 8. Auflage i 856, S. 73.

I6 Ausführlich dazu Knieper, Gesetz und Geschichte, I996, S. i I 5 ff und S. 67 ff.

I7 Vgl. nur Medicus, Abschied von der Privatautonomie im Schuldrecht?, I994, S. 2 I ff, $32 \mathrm{ff}$. 
stimmten Personenkreis nicht mehr als Einladung zum Vertragsangebot, als klassische invitatio ad offerendum qualifiziere, sondern selbst bereits als bindendes Angebot. ${ }^{18}$ Hier tut das Gesetz in Wahrheit nichts weiter als die Bindungswirkung einer Willenserklärung nach vorn zu verschieben.

Das dritte Beispiel betrifft die dem klassischen römischen Recht unbekannte, inzwischen aber vollständig triviale Rechtsfigur der Stellvertretung. Sie ist aus keinem modernen Wirtschaftssystem wegzudenken, hat aber der juristischen Dogmatik außerordentliche Schwierigkeiten bereitet, die bis zur Kennzeichnung als »juristisches Wunder « oder »Monstrosität « reichen. ${ }^{19}$ Wenn es tatsächlich stimmte, dass alles Recht auf die im menschlichen Willen ausgedrückte Selbstbestimmung gründet, wäre es allerdings wirklich kaum erklärbar, wie die Erklärung eines Menschen »unmittelbar für und gegen den Vertretenen « wirken kann, wie es $\$ I64 BGB nicht anders als alle anderen Gesetze bestimmt, welche die Stellvertretung regeln. Wiederum ist zu Fiktionen und haltlosen OrganSimulationen gegriffen worden, um die Bindungswirkung für Dritte zu begründen, anstatt die ebenso banale wie naheliegende Erklärung anzunehmen, das Handeln in fremdem Namen als Tatsache anzunehmen, an die das Gesetz eine Rechtswirkung für die dritte Person knüpft.

Die Beispiele belegen insgesamt, dass eine Erfassung zivilrechtlicher Figuren ohne Rekurs auf das Gesetz nicht auskommt. Weder die Vernunft noch der Wille schaffen durchsetzungsfähige Rechte und korrespondierende Pflichten, sondern das Gesetz und der richterliche Befehl. Die Vordenker der großen Kodifikationen wussten das, wenn sie bei aller Betonung der Willensfreiheit darauf bestanden, dass der Wille zur Willensmacht nur werden kann, weil sie »von der Rechtsordnung verliehen ist. $^{20}$

In dieser Perspektive verlieren im Übrigen auch die oben angeführten Angriffe auf das Vertragsrecht ihren Schrecken, die eine Determiniertheit des Willens behaupten, gleichgültig ob aus Gründen neurologischer Verstrickung oder ökonomischer Zwänge. Die Rechtsgeschäftslehre und die Zivilgesetzbücher haben stets und zu Recht darauf bestanden, dass die Gültigkeit einer Erklärung nicht durch Motive, Erwartungen, Wünsche und Kalküle belastet werden kann. Der »Motivirrtum ${ }^{2 \mathrm{II}}$ gibt niemals das Recht, die Verbindlichkeit eines Engagements aufzulösen. Es ist für das Zivilrecht selbstverständlich, dass der Kunsthändler, der auf den Vertragsschluss existentiell angewiesen ist, sich nicht von seiner Verpflichtung mit der Begründung lösen kann, dass seine ökonomischen Erwartungen enttäuscht worden sind. Ebenso wenig kann das Gutachten eines Hirnforschers, das einem Vertragspartner bestätigt, er habe nicht frei entschieden, sondern einem neurologischen Reflex nachgegeben, zur Unverbindlichkeit seiner Willenserklärung führen. Auch die Ungleichverteilung der Marktmacht, die im Prinzip für jeden, der an eine substantiell gehaltvolle Willensfreiheit glaubt, unerträglich sein müsste, führt nicht zur Auflösung der Vertragsverbindlichkeit, sondern bestenfalls zu sozialstaatlicher Kompensation. Die Freiheit des Willens sind ebenso wie seine Verbindlichkeit normative Annahmen. Sie gelten gemäß dem Gesetz, weil und solange sie sich in einer subjektiven und objektiven

I 8 So zum Beispiel D. Hart, Soziale Steuerung durch Vertragsabschlusskontrolle, in: Kritische Vierteljahresschrift I986, S. 2 I I ff.

I9 Vgl. besonders die Untersuchungen von Müller-Freienfels, Stellvertretungsregelungen in Einheit und Vielfalt, 1982.

20 Vgl. statt aller: Windscheid (Fn. 6), S. 99.

2 I Vgl. dazu etwa Palandt/Heinrichs, BGB, 66. Aufl. 2007, \ I I9 Rdnr. 29. 
Normalität bewegen, wobei auch die Definition der subjektiven Normalität keine anthropologische ist, sondern wiederum normativ festgelegt wird.

\section{Die Freibeit der Person}

Diese Aussagen führen unweigerlich zum Subjekt, genauer: zum Rechtssubjekt. Wer ist das, von dem rechtserhebliche Handlungen, Rechtsgeschäfte, Willenserklärungen, Vertragsangebote und Delikte ausgehen, wem ordnet das Recht sie zu? Auf einer neuen Ebene wiederholen sich die bereits im Rahmen der Willensfreiheit diskutierten konstruktiven Probleme. Dort wie hier unterscheidet die juristische Dogmatik nicht scharf genug zwischen einer anthropologisch-philosophischen Konstruktion des Menschen und einer juristischen Konstruktion der Person. Das wäre nicht weiter bemerkenswert, wenn die Vermischungen nicht zu großen Schwierigkeiten bei der Erfassung der juristischen Person führten erneut eine Rechtsfigur, an deren praktischer Notwendigkeit seit Jahrhunderten kein Zweifel besteht, die theoretisch zu erfassen aber zu weltfremden Konstruktionen führt, solange die bürgerliche Rechtssubjektivität im Prinzip beim Menschen verortet wird.

An anderer Stelle habe ich ausführlich die »Theorien « dargestellt, die versuchen, die juristische Person in den konstruktiven Griff zu bekommen: ${ }^{22}$ Die einen behaupten, es handele sich um Fiktionen des Menschen, eine immerhin wenig befriedigende Aussage angesichts globaler Wirtschaftsbeziehungen, in denen der weitaus größte Teil der Verträge durch Gesellschaften, d.h. nach dieser Auffassung durch fiktive Einheiten abgewickelt wird. Die anderen behaupten gegen alle Anschauung, bei den juristischen Personen handele es sich um »reale Verbandspersönlichkeiten«, also um eine Art Menschen, denen entsprechend heute eher verschämt - quasi-biologische Organe angedichtet werden.

Beide Konstruktionen verkennen, dass es im bürgerlichen und Wirtschaftsrecht nicht um Menschen geht, sondern von vornherein um Personen, denen das Gesetz Rechtspersönlichkeit und -subjektivität zuerkennt. Insofern genießen die Menschen als physische Personen den Status der juristischen Persönlichkeit. Dies ist weder eine dogmatische Spielerei noch gar Ausdruck anti-humanistischer Kälte. Das Gegenteil wird offensichtlich, wenn man bedenkt, dass die staatliche Zuerkennung der vollständigen und gleichartigen Rechtssubjektivität für alle Menschen als große rechtspolitische und zivilisatorische Leistung angesehen werden kann, mit der vom i 8. Jahrhundert an zunächst in Europa und zunächst beschränkt auf men of ripe years and of sound mind die »Fähigkeit, Träger von Rechten und Pflichten « zu sein, auf alle Menschen unabhängig von Geschlecht, Rasse, Religionszugehörigkeit oder neurologischer Determiniertheit ausgedehnt worden ist. Religiöse, ethnische oder klassenorientierte Diskriminierungen wie im Corpus Iuris Canonici, dem Entwurf eines faschistischen Volksgesetzbuches, oder im sowjetischen Zivilgesetzbuch haben sich auf die Dauer nicht halten können. Noch heute aber ist diese bürgerlich-rechtliche Grundbestimmung keineswegs weltweit selbstverständlich, sondern immer wieder einmal partiellen Aushebelungen ausgesetzt. 
Aus dem Gesagten ergibt sich hoffentlich mit einiger Klarheit, dass die Konzeption der Freiheit des Willens und der Rechtspersönlichkeit nicht gelöst von den historischen Entstehungsbedingungen verstanden werden kann. Sie ist in Europa entstanden in Gegnerschaft zur feudalen und ständischen Gesellschaftsordnung, deren Recht den individuellen Willen eben nicht als frei, sondern als vielfältig gebunden definiert sowie eine klare Hierarchie und Relativität der Rechtssubjektivität der Menschen festgelegt hatte. Die Orientierung war (rechts-)philosophisch vorgedacht; für die Zeitgenossen gab es jedoch keinen Zweifel, dass sie ohne staatliches Gesetz nicht Realität werden konnte. Angesichts seiner praktischen Verbreitung mag diese Einsicht heute in den theoretischen Hintergrund rücken. Sie bleibt dennoch nicht weniger grundlegend, was nicht zuletzt immer dann wieder schmerzlich in den Vordergrund rückt, wenn diese oder jene politische, religiöse oder sonst wie ideologische Bewegung die Rechtssubjektivität bestimmter Menschengruppen einschränkt oder ausschließt. Es ist spätestens an dieser Stelle nötig, eine Qualifikation des Freiheitsbegriffs einzuführen, die für seine historische Kraft und sein rechtsphilosophisches Verständnis ebenso grundlegend ist wie für moderne Auflösungserscheinungen. Die Aufklärungsphilosophie hatte zur normativen Basis die Überzeugung, dass der Mensch im gesellschaftlichen Leben nicht seinen Begierden, Trieben und Leidenschaften nachgebe, sondern vernünftig handele. Die vernunftrechtliche Variante des Naturrechts lebt von dieser Überzeugung. Frei ist der Mensch erst als sittliche, selbst-beherrschte Persönlichkeit, gereinigt von der Willkür der Affekte und Spontaneitäten. Die Person erkennt und anerkennt die Verbindlichkeit des Rechts in Vernunft. Ethisches und juristisches Handeln, subjektives und objektives Recht, Freiheit und Verbindlichkeit decken sich. Der Handelnde und seine Willensäußerungen sind frei nur, wenn und soweit das allgemeine Gesetz als das eigene anerkannt ist: »Der sittliche Mensch begehrt die Freiheit, weil er das Gute aus eigenem Antrieb zu tun wünscht «. ${ }^{23}$ Dieses Zitat ist symptomatisch und steht für ähnliche Aussagen bei Kant, Hegel, Savigny und anderen Rechtsphilosophen und -wissenschaftlern der Aufklärung. ${ }^{24}$ Die vernünftige Freiheit ist - man ist versucht zu sagen: >natürlich - gesellschaftlich normiert. Sie bewährt sich in der Nützlichkeit des Erwerbs, der Produktion, der Eigentumsmehrung. Sie wehrt ab, was den Menschen »das Fieber in den Kopf treibt «, und unterdrückt »unproduktive Verausgabung «. ${ }^{25}$

Es ist schwer, sich ein Bild $\mathrm{zu}$ machen und nachzuvollziehen, in welchem Umfang diese normativen Aussagen zur Freiheit der Person und seines Willens jemals eine empirische Entsprechung in der europäischen oder außereuropäischen gesellschaftlichen Realität gekannt haben. Jedoch ist nicht zu verkennen, dass die Erziehungen und Zurichtungen jedenfalls des Knaben in der um das Eigentum der Väter zentrierten patriarchalischen Familie eben diese Form der Vernunft herzustellen versucht haben. Das war schon deshalb sinnhaft, weil ihre jeweilige Existenz von der individuellen Leistungsbereitschaft und Triebbeherrschung, kurz: >Vernünftigkeit< der männlichen Erben - ebenso wie von der anders zustande kommenden Mütterlichkeit der Frauen - abhing. ${ }^{26}$ Außerdem

23 So das Zitat bei von Jhering, Geist des römischen Rechts auf den verschiedenen Stufen seiner Entwicklung, I 852 , Band II, I, S. I 3 I f.

24 Das kann ich hier im Einzelnen nicht ausführen und verweise deshalb auf Knieper (Fn. I6), S. 68 ff.

25 So Bataille, Die Aufhebung der Ökonomie, 1946, S. Io ff. 
ist wahrscheinlich, dass die tiefen Spuren einer kollektiven Disziplinierung und Konditionierung, welche die Jahrhunderte langen Folterungen und Scheiterhaufen der christlichen Inquisition in das Gedächtnis der Menschen eingegraben hatten, als internalisierte soziale Kontrollinstanz weiterwirkten. Es waren ja die Philosophen der Aufklärung gewesen, die gegen die Inquisition und das Strafrecht der frühen Neuzeit angeschrieben hatten, nicht zuletzt mit dem Argument einer intrinsischen Sittlichkeit und Vernünftigkeit des Menschen. ${ }^{27}$

Wie dem auch sei, es bestehen für mich wenige Zweifel daran, dass der aufklärerische und pandektistische Freiheitsbegriff nicht ohne seine Begrenzung und Konditionierung durch Vernunft und Sittlichkeit verstanden werden kann. Wenn in der heutigen zivilrechtlichen Dogmatik der ernste und sittliche Ton der Pandektistik in postmodernem Duktus hedonistisch aufgelockert wird, wenn darauf bestanden wird, endlich Subjektivität zuzulassen und die »Vielfalt der Affekte, der ästhetischen, ökonomischen, emotionalitätsbezogenen etc. Interessen ${ }^{28}$ im Zivilrecht zu berücksichtigen, dann handelt es sich nicht um eine Fortentwicklung der Aufklärungsphilosophie, sondern um ihre Karikierung.

Mir scheint auch - und ich betone die Subjektivität der Anschauung -, dass bei aller Betonung des Hedonistischen die externe soziale Kontrolle und die Normierung menschlichen Verhaltens unaufhaltsam zunimmt, und dies, je weniger die patriarchalische >Menschenproduktion< gewährleistet ist. Das geschieht bisweilen unter dem Eindruck und Vorwand der Bekämpfung einer Bedrohung oder Krise wie des Rauschgiftkonsums, des Missbrauchs oder der Vernachlässigung von Kindern, eines Terroranschlags in New York oder anderswo, überwiegend aber unter dem sogenannten >Zwang der Verhältnisse< der Massenproduktion, des fast food, des Massentourismus, der Massenmode und anderer Uniformisierungen, die sich spontan zu entwickeln und nach ebenso normierten sozialen Lösungen wie Massenrecht zu verlangen scheinen. Individuelle Willensäußerungen stören in diesen Arrangements. Mir scheint ebenso, dass je mehr die Unausweichlichkeit dieser Entwicklung zur Gleichförmigkeit propagiert wird, umso mehr in praktisch inhaltsloser Weise der hohe Wert der individuellen Freiheit gepredigt wird.

Solche Entwicklungen gehen am Vertragsrecht nicht vorbei. Das Spektrum reicht von der Zunahme zwingenden Rechts in sSonderprivatrechten< für Verbraucher, für Händler, für Heranwachsende und andere, auf deren Selbstdisziplin kein Verlass zu sein scheint, aber auch im sogenannten selbstgeschaffenen Recht der Wirtschaft, mit dem massenhaft Risiken zum eigenen Vorteil umverteilt werden, bis hin zu halbstaatlichen Prinzipien der good corporate governance, die allzu gierigen Managern eine Leine der Vernunft anlegen sollen.

Je offensichtlicher aber die Formulierung eines individuellen Willens im Massenvertrag, in Allgemeinen Geschäftsbedingungen, in Standardformularen zurücktritt, desto offensichtlicher wird, dass die immer schon benannte Dichotomie des Schuldrechts nicht vertragliche von gesetzlichen Schuldverhältnissen trennt, also solche, von denen gesagt wird, dass in ihnen der Wille beziehungsweise das Gesetz regieren. Tatsächlich sind alle Schuldverhältnisse sgesetzlicher. Die Dichotomie liegt denn auch nicht in der Rechtsbegründung, sondern in der

26 Diese Zusammenhänge haben wir ausführlich dargestellt in Heinsohn/Knieper, Theorie des Familienrechts, 2. Aufl. 1976, bes. S. 3i ff.

27 Wiederum verweise ich auf unsere ausführlichere Analyse dieser Zusammenhänge in Heinsohn/Knieper/ Steiger, Menschenproduktion - Allgemeine Bevölkerungslehre der Neuzeit, I979, bes. S. 46 ff.

28 So zum Beispiel Struck, Juristische Schulung, I993, S. 992 ff/999; ebenso Williamson, Die ökonomischen Institutionen des Kapitalismus, I990, S. 25 ff. 
Rechtsfolge: Gewisse Schuldverhältnisse werden so konstruiert, dass sie eine Verpflichtung zu monetärer Kompensation und Schadensersatz begründen, während andere auf der Verpflichtung zur Primärleistung bestehen. Grob gesprochen verlaufen diese Trennungen parallel zu den Konstruktionen ,Delikt' und >Vertrag<. Die Rechtsprechung und Wissenschaft haben es aber nicht an Phantasie mangeln lassen, zugunsten des Grundsatzes des favor contractus Verträge auch dort zu konstruieren, wo kein Wille geäußert wurde. Man denke nur an die selbstverständlich hingenommenen sogenannten sfaktischen Vertragsverhältnisse ${ }^{29}$

Es bleibt das Eigentum. ${ }^{30}$ Seine Behandlung eignet sich vorzüglich, um die hier vorgestellten Einbettungen der Freiheit erneut zu verifizieren, nämlich:

- die antifeudale Stoßrichtung,

- die staatlich-rechtliche Garantie,

- die implizite Annahme eines vernünftigen Gebrauchs.

Noch das I8I I in der Monarchie erlassene österreichische ABGB kennt eine Aufspaltung des Eigentums in der Weise, dass es nicht nur ein vollständiges Eigentumsrecht, sondern auch einen »Obereigentümer « und einen »Nutzungseigentümer« soll geben können $\left(\mathbb{S} \int_{357 / 359} \mathrm{ABGB}\right)$, während der der bürgerlichen Revolution verpflichtete französische Code Civil bereits I 804 diese der feudalen Eigentumsordnung verpflichtete Unterteilung aufgegeben und bestimmt hatte, dass jedermann mit dem Eigentum das Recht erhalte, mit der Sache nach Belieben zu verfahren, sie zu nutzen oder über sie zu verfügen (Art. 544 CC). In Deutschland konnte sich der Gesetzgeber wieder einmal auf die rechtsphilosophischen Vorüberlegungen von $\mathrm{Kant}^{3 \mathrm{I}}$ und Hegel ${ }^{32}$ stützen, die beide die feudalen Eigentumsformen und -beschränkungen als überholt abgelehnt hatten. Der Gesetzgeber erklärte, dass es sich bei den zur bloßen Nutzung berechtigenden Lehen, also das Nutzungseigentum oder auch dominium utile, um Einrichtungen handele, »welche einer längst vergangenen Gestaltung der politischen und wirtschaftlichen Verhältnisse ihre Entstehung verdanken ... Sie sind dem Absterben verfallen und deshalb zur Aufnahme in die bürgerliche Gesetzgebung nicht geeignet «.33 So geschah es, dass in Deutschland ein einheitlicher und umfassender Eigentumsbegriff akzeptiert wurde $(\mathbb{S} 903 \mathrm{BGB})$. Die Monarchie hatte sich den rechtspolitischen Forderungen der bürgerlichen Bewegungen gebeugt.

Damit hatte sich nicht endlich ein zeitloses Naturrecht realisiert, wie es an manchen Literaturstellen heißt. 34 Vielmehr war und ist das Eigentumsrecht begründet, weil und soweit es das staatliche Gesetz einrichtet, garantiert und schützt. Überraschend klar hatte bereits Savigny befunden: »Alles Recht erhält seine Realität und Vollendung erst im Staate, und so konnte auch das Eigentum zu einem wirklichen Daseyn nur dadurch gelangen, dass es zunächst auf den Staat und vermittelst der im positiven Recht des Staates ausgebildeten Regeln auf die einzelnen Rechtsgenossen im Staate, als Eigentümer, bezogen wurde«.35 Dieser Aussage ist bis heute wenig hinzuzufügen.

29 Vgl. statt aller Palandt/Heinrichs (Fn. 2I), Einführung vor $\ 145$ Rdnr. 25 ff.

30 Für eine ausführlichere Argumentation verweise ich wiederum auf Knieper (Fn. I6), bes. S. I 99 ff.

3I Kant, Metaphysik (Fn. I3), S. 382.

32 Hegel, Grundlinien (Fn. I I), $\int \$ 6_{2} / 6_{3}$.

33 Motive zu dem Entwurfe eines Bürgerlichen Gesetzbuches für das Deutsche Reich, I888, Band III, S. 6.

34 Dazu ausführlich und mit Hinweisen zur Literatur Knieper (Fn. I6), S. 20 I ff.

35 Savigny (Fn. 5), S. 374 . 
Welche politische Brisanz die umfassende Eigentumsgarantie hat, zeigt sich daran, dass der Gesetzgeber sie auf Sachen beschränkt hat. Die Immaterialgüterrechte, das ,intellektuelle Eigentum' war nicht erfasst, obwohl die großen internationalen Konventionen und die nationalen Gesetze zu den Patenten und anderen Rechten zum Zeitpunkt der Verabschiedung des BGB bereits in Kraft waren. Ich deute diese Beschränkung nicht als Ignoranz des Gesetzgebers gegenüber modernen Tendenzen, sondern als bewusste Entscheidung, den umfassenden Schutz - noch in der Abwehr feudaler Verhältnisse - nur bei Sachen zuzulassen, während das intellektuelle Eigentum von vornherein mit Beschränkungen der Dauer, des Inhalts und des Nutzungsumfangs geschaffen wurde.

Diese Überlegung führt zu der Frage, ob tatsächlich das Eigentumsrecht so umfassend und unbeschränkt ist und gedacht wurde, wie es im antifeudalen Reflex formuliert worden ist. Wir kommen noch einmal auf unser Eingangsbeispiel zurück: Kann der Eigentümer sein Recht wirklich brauchen und missbrauchen, kann er fruchtbares Land brach liegen oder durch vermeidbare Umweltverschmutzung wertlos werden, ein Wohnhaus leer stehen, ein florierendes Unternehmen liquidieren lassen, kann er mit einem Wort im ökonomischen oder ökologischen Sinne unvernünftig mit seinen Sachen umgehen?

Immer wieder haben Autoren aus den verschiedensten kulturellen und ideologischen Lagern - links und rechts, römisch- und deutschrechtlich, sozialistisch und faschistisch, religiös und atheistisch, romantisch und utilitaristisch - gegen diese Konsequenzen resignierte Ablehnung geäußert. Sie haben gemahnt, dass es »kein absolutes, das heißt der Rücksicht auf die Gemeinschaft entbundenes Eigentum « geben dürfe, ${ }^{36}$ und geschimpft, dass es »ein kulturfeindlicher Widersinn«sei, dass »ein Stück unseres Planeten einem einzelnen Menschen in derselben Weise eigen sein soll wie ein Regenschirm oder ein Guldenzettel «,37 dass »Brote nicht verfüttert, sondern gegessen werden, Bücher und Bilder gelesen und zur Betrachtung aufgehängt, nicht als Kapitalanlage gestapelt werden, Ware umgesetzt und nicht gelagert werden « solle. $3^{8}$

Die moralischen Bedenken scheinen sich an den als umfassend vorgestellten Eigentümer-Befugnissen und den entsprechenden Gesetzesformulierungen zu brechen. So fasst der französische Rechtssoziologe Duguit eine auch heute noch herrschende Meinung zusammen, wenn er aus der Formulierung des Art. 544 Code Civil folgert, dass der Eigentümer »sein Land unkultiviert, seine Stadtgrundstücke unbebaut, seine Häuser unvermietet und ohne Unterhaltung, sein mobiliares Kapital unproduktiv lassen« dürfe.39

Ist das so? Ist diese Interpretation durch den Wortlaut der Gesetze, durch ihren historischen Kontext, ihren Systemzusammenhang und ihre Teleologie begründet? Ich meine: nein. Zunächst fällt nach einer ganz oberflächlichen Lektüre aller Gesetze, vom Code Civil über das BGB bis hin zum neuen niederländischen Wetboek von 1992 (Art. I - 5.I.2) und dem Code Civil Quebecs von I994 (Art. 947) auf, dass die Eigentümer-Rechte positiv als Nutzen, Besitzen und Verfügen definiert werden und von negativen Befugnissen wie dem Missbrauch, dem Nichtgebrauch, der Zerstörung nirgendwo die Rede ist. Dies ist meines Erachtens keine begriffsjuristische Spielerei. Vielmehr bettet sich diese Lektüre so zwanglos überzeugend in den rechtshistorischen und rechtsphilosophischen Zusammenhang der Zivilgesetzbücher ein, dass im Gegenteil das Hineinlesen 
der Negativbefugnisse durch die herrschende Rechtswissenschaft nur als eilfertige Verbeugung vor der Macht interpretiert werden kann.

Wie schon gesagt, erklärt sich die gesetzliche Fassung und Betonung der Freiheit des Eigentums an Sachen aus seiner antifeudalen Stoßrichtung. Der Begriff der Freiheit aber ist getragen von eben den philosophischen Überzeugungen und juristischen Vorverständnissen, welche auch die Freiheit der Person und des Willens tragen. Der in die bürgerliche Freiheit entlassene Mensch ist »im Eigentum als Vernunft«, hatte Hegel proklamieren können. ${ }^{\circ}$ Das Rechtssubjekt ist normativ konzipiert als physische oder juristische Person, die einem ,natürlichen' zweckrationalen Kalkül folgt und sein Vermögen durch legalen Gebrauch, Nutzung und Verfügung, durch Produktion und Handel mehren werde. Der Verschwender, der Exzentriker, der Bösartige, der ein a-normales Vergnügen daran hat, sein Eigentum zum Schaden anderer einzusetzen oder zu entziehen, ohne selbst davon einen materiellen Vorteil zu haben, der östliche, westliche und südliche Oligarch, der seine Betriebsgewinne in Schlösser, Bildersammlungen, Prinzessinnen, Models und Prostituierte steckt, anstatt es in die Betriebe zu deren langfristiger Erhaltung zu re-investieren, kann damit vielleicht angesichts einer willfährigen Justiz davonkommen. Auf das in der Aufklärung formulierte Konzept der Freiheit des Eigentums und ihren Schutz kann er sich nicht berufen. Anders herum: Wenn ein Eigentümer gegen seinen unvernünftigen Willen dazu verpflichtet wird, sein Vermögen zu erhalten und bei angemessener Rentabilität zu nutzen, wozu ihn die bürgerliche Vernunft ohnehin bewegen müsste, also zur Vermietung seiner nicht eigen-genutzten Wohnungen, zur produktiven Anlage seines Kapitals, zur Bebauung seiner Felder, dann ist das zivilrechtlich kein Eingriff in die positiven Eigentümerbefugnisse, keine Konfiskation und keine Enteignung, sondern der Vollzug der Vernunft, die sich individuell selbstkontrolliert nicht herstellt.

Der Refrain wiederholt sich: Die philosophisch entwickelte und rechtlich gefasste Freiheit des Eigentums als eine der großen Freiheiten der bürgerlichen Gesellschaft ist alles andere als Beliebigkeit und Spaß, sondern eingebettet und orientiert in normativ definierte Vernunft, die sich entweder selbst, im Rechtssubjekt bildet und äußert oder als »Idee der Gesellschaft $\ll^{\mathrm{I}}$ durchgesetzt werden muss. Dass dies keine utopischen oder gar anti-naturrechtlichen Spinnereien sind, dokumentiert das Recht des intellektuellen Eigentums, wo gesetzliche Orientierungen und Beschränkungen selbstverständliches positives Recht sind.

\section{Summary}

Die Freibeit oder Unfreibeit des Willens sind keine anthropologischen oder neurologischen Konstanten, sondern normative Annahmen, deren Äußerungen vom Privatrecht als Tatsache qualifiziert werden. Den im Kampf gegen die Ständegesellschaft durchgesetzten Freiheiten des Vertrages, der Person und des Eigentums unterliegen auch diese Annabmen. Deren Zerbröckeln wird dazu fübren, dass die bürgerlich - rechtliche Konzeption der Freiheiten fundamental in Frage gestellt wird, was sich bereits heute in weitgehender und zunehmender sozialer Kontrolle praktisch durchsetzt. 